
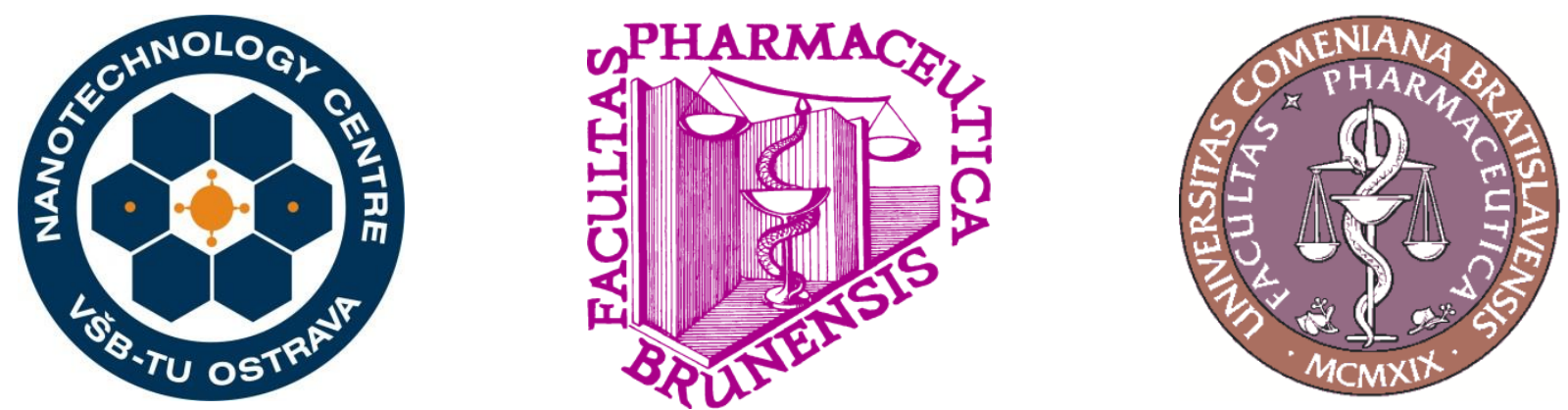

\title{
Preparation of Risedronate Nanoparticles Using Selected Polymeric Excipients
}

\author{
Eliska Vaculikova ${ }^{1,2}$, Daniela Placha $^{2}$, Martin Pisarcik ${ }^{3}$ and Josef Jampilek ${ }^{1 *}$ \\ ${ }^{1}$ Department of Chemical Drugs, Faculty of Pharmacy, University of Veterinary and \\ Pharmaceutical Sciences, Palackeho 1/3, 61242 Brno, Czech Republic; e-mail: \\ josef.jampilek@gmail.com \\ ${ }^{2}$ Nanotechnology Centre, VSB - Technical University of Ostrava, 17. listopadu 15/2172, \\ 70833 Ostrava - Poruba, Czech Republic \\ ${ }^{3}$ Department of Chemical Theory of Drugs, Faculty of Pharmacy, Komensky University, \\ Ulica Odbojarov 10, 832 32, Bratislava 3, Slovakia \\ * Authors to whom correspondence should be addressed.
}

\begin{abstract}
Almost all newly designed drugs (new chemical entities) demonstrate problematic aqueous solubility and/or permeability through biological membranes, i.e. they show poor bioavailability. One of the progressive ways for increasing bioavailability is a technique of nanoparticles preparation, which allows many drugs to reach the site of action. Risedronate sodium (Biopharmaceutical Classification System class III) was chosen as a model compound with high solubility and low permeability. Nanoparticles of risedronate sodium are stabilized by polyethylene glycol or carboxymethyl dextran sodium salt. All prepared samples were measured by dynamic light scattering, and it was found that the particle size ranged from 2.8 to $9.1 \mathrm{~nm}$.
\end{abstract}

Keywords: Bisphosphonates; Risedronate; Nanoparticles; Polymeric excipients; Dynamic light scattering.

\section{INTRODUCTION}

Bisphosphonates (BPs) such as the pyrophosphate analogues (see a general structure in Figure 1) are a group of drugs that are widely used in practice. They are the most effective bone resorption inhibitors currently available for treatment of Paget's disease, tumourassociated bone disease and osteoporosis. BPs contain a P-C-P backbone with two covalently bound side chains by which BPs differ from each other. The ability of BPs to inhibit bone resorption depends on the presence of two phosphonate groups in the P-C-P structure, which appears to be required for interaction with a molecular target in the osteoclast as well as for binding bone mineral [1-3]. Side chains modify physico-chemical properties of molecules and intensity of interaction with osteoclast, i.e. they are important for antiresorptive potency 
of these drugs. The mechanism of action of BPs is induction of apoptosis in osteoclasts [4]. Osteoclasts are multinuclear cells; they are derived from hematopoietic stem cells. They dissolve mineral and degrade organic bone matrix [5].

There are several injectable BPs: etidronate (Didronel ${ }^{\circledR}$ ), pamidronate (Aredia ${ }^{\circledR}$ ) and zoledronate $\left(\right.$ Zometa $^{\circledR}$ ), which may be administered every three months or yearly. Per oral BPs alendronate $\left(\right.$ Fosamax $\left.^{\circledR}\right)$ and risedronate $\left(\right.$ Actonel $\left.^{\circledR}\right)$ are taken daily, weekly or monthly; and ibandronate $\left(\right.$ Boniva $\left.^{\circledR}\right)$ is approved to be taken monthly. Oral bioavailability of BPs is very low (in most cases lower than 3\%) due to their high hydrophilicity. Pharmacokinetics of BPs is better when they are administered on an empty stomach. Food rich in calcium or iron, antacids, mineral water and coffee can lower their availability. BPs are not metabolised in the human body; they are so-called hard drugs; there is no any enzyme in the human body to metabolise them. They are absorbed in the stomach and intestine [6].

Risedronate (risedronate monosodium salt, systematic name: sodium 1-hydroxy-1phosphono-2-(pyridin-3-yl-ethyl)phosphonate), see Figure 1, is a member of class III of the Biopharmaceutics Classification System and belongs to generation III of BPs. It has a chemically unique component as compared with other BPs, which is believed to reduce the likelihood of gastro-intestinal side effects. Risedronate is more potent in blocking the dissolution of bone than etidronate and alendronate [7,8]. It has good solubility in water, but its permeability is really poor [9]. A clinical trial focused on an availability of risedronate after oral intake showed that absolute bioavailability of risedronate after oral administration is less than 1\%. Bioavailability of tablet formulation and oral solution is equivalent [10]. Nevertheless oral route is the preferred one in case of chronic bisphosphonate therapy.

Figure 1. Structures of bisphosphonates used in practice.
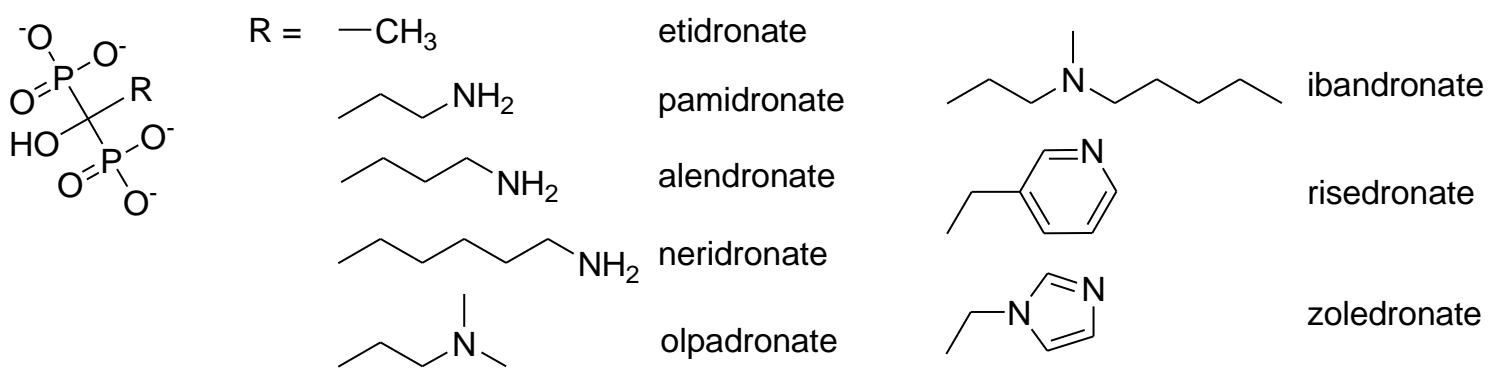

Generally strategies/structural modifications to improve permeability are based on a few fundamental concepts: reduction of ionizability, increase of lipophilicity, reduction of polarity or reduction of hydrogen bond donors or acceptors. Formulation is other strategy for improving permeability and bioavailability; for example, permeability enhancers, surfactants or pharmaceutical complexing agents can be used in the oral dosage form [11].

New formulations of bisphosphonates are developed with a view to increase their oral bioavailability and reduce side effects. For example, ethylenediaminetetraacetic acid (EDTA) in formulations with BPs enhanced their absorption in intestine. However further investigation was not possible because of the damaging effect of the chelator concentration necessary for the absorption enhancement on mucosal integrity [12]. Another strategy was preparation of peptide prodrugs that can be recognized by the intestine carrier system and subsequently transported. Also titanium dioxide particles were prepared for the controlled release system of risedronate [12]. Other approach to modification of risedronate properties was preparation of co-crystals [13].

The problem of poor permeability can be also solved by preparation of nanoparticles. In general, nanoparticles for systemic applications should range from 10 to $100 \mathrm{~nm}$, with minimum surface charge. Nanosize allows effective systemic circulation and enables many 
pharmacological agents to reach the site of action that is not available for larger particles. Nanoparticles can be used for permeation through blood brain barrier [14-16].

This contribution is a follow-up paper to our previous studies [13,17,18] and deals with preparation of nanoparticles of risedronate monosodium salt. Nanoparticles are stabilized by polyethylene glycol (macrogol) or carboxymethyl dextran sodium salt - excipients selected based on the GRAS (Generally Recognized as Safe) list, which means that they are not toxic for human body. The excipients were applied in various concentrations.

\section{RESULTS AND DISCUSSION}

Based on pilot screening [17,18], two polymeric excipients such as polyethylene glycol 6000 (sample series 1) and sodium salt of carboxymethyl dextran (sample series 2) were used in this investigation. Three water solutions were prepared from excipients with mass concentration of $1 \%$ (samples a), 3\% (samples b) and 5\% (samples $\mathbf{c}$ ). As risedronate sodium salt is soluble in water, this medium was chosen as a solvent. Risedronate sodium was solved in water, added to the solution of excipient under continuous stirring. Then ultrasonic bath was used for destruction of possible agglomeration, and finally the solvent was evaporated to the third of the initial volume.

All prepared samples were measured by dynamic light scattering [19]. The particle size distribution is presented in Table 1; the particle size range was narrow. In detail this fact is also shown in Figure 2, where the dependence of particle size [nm] expressed as mean $\pm \mathrm{SD}$ of risedronate sodium on the concentration [\%] of both excipients in water is illustrated. From particle size measurement it can be seen that nanoparticles under 100 nanometres were found in all the samples, nevertheless carboxymethyl dextran sodium salt provided slightly less nanoparticles (range 2.8-3.5 nm) in comparison with macrogol (particle size ranged 7.7-9.1 nm). According to the results (see Table 1), no influence of the concentrations of excipients in the range $1-3 \%$ on particle size was observed.

Table 1. Particle size $[\mathrm{nm}]$ expressed as mean $\pm \mathrm{SD}(n=5$ independent measurements) of risedronate particles and concentration [\%] of macrogol 6000 (PEG) and sodium salt of carboxymethyl dextran (SCMD) in water.

\begin{tabular}{|c|c|c|}
\hline Sample & Excipient/concentration[\%] & Particle size [nm] \\
\hline $\mathbf{1 a}$ & $\mathrm{PEG} / 1$ & $9.1 \pm 1.9$ \\
\hline $\mathbf{1 b}$ & $\mathrm{PEG} / 3$ & $8.2 \pm 1.1$ \\
\hline $\mathbf{1 c}$ & $\mathrm{PEG} / 5$ & $7.7 \pm 2.5$ \\
\hline $\mathbf{2 a}$ & $\mathrm{SCMD} / 1$ & $3.5 \pm 1.1$ \\
\hline $\mathbf{2 b}$ & $\mathrm{SCMD} / 3$ & $2.8 \pm 0.4$ \\
\hline $\mathbf{2 c}$ & $\mathrm{SCMD} / 5$ & $3.8 \pm 1.2$ \\
\hline
\end{tabular}

The dispersity is a measure/degree of the homogeneity/heterogeneity of sizes of particles in a mixture/system. It is possible to see this feature on the width of the particle-size distribution, which is described as standard deviations (SDs), see Table 1. It is possible to suppose that the investigated nanoparticles are spherical, because the size in dynamic light scattering means the hydrodynamic diameter of the particle. All samples were dispersed by ultrasonics directly before the measurement to avoid possible re-agglomeration. Stabilization of the dispersed samples was achieved by surfactants and by the constant temperature. The measuring cell was 
equilibrated at $25^{\circ} \mathrm{C}$, so the Brownian motion of nanoparticles was influenced by their size only.

Figure 2. Dependence of particle size [nm] of risedronat sodium on concentration [\%] of macrogol 6000 (PEG) and sodium carboxymethyl dextran (SCMD) in water. Particle size is expressed as mean $\pm \mathrm{SD}$ ( $n=5$ independent measurements).

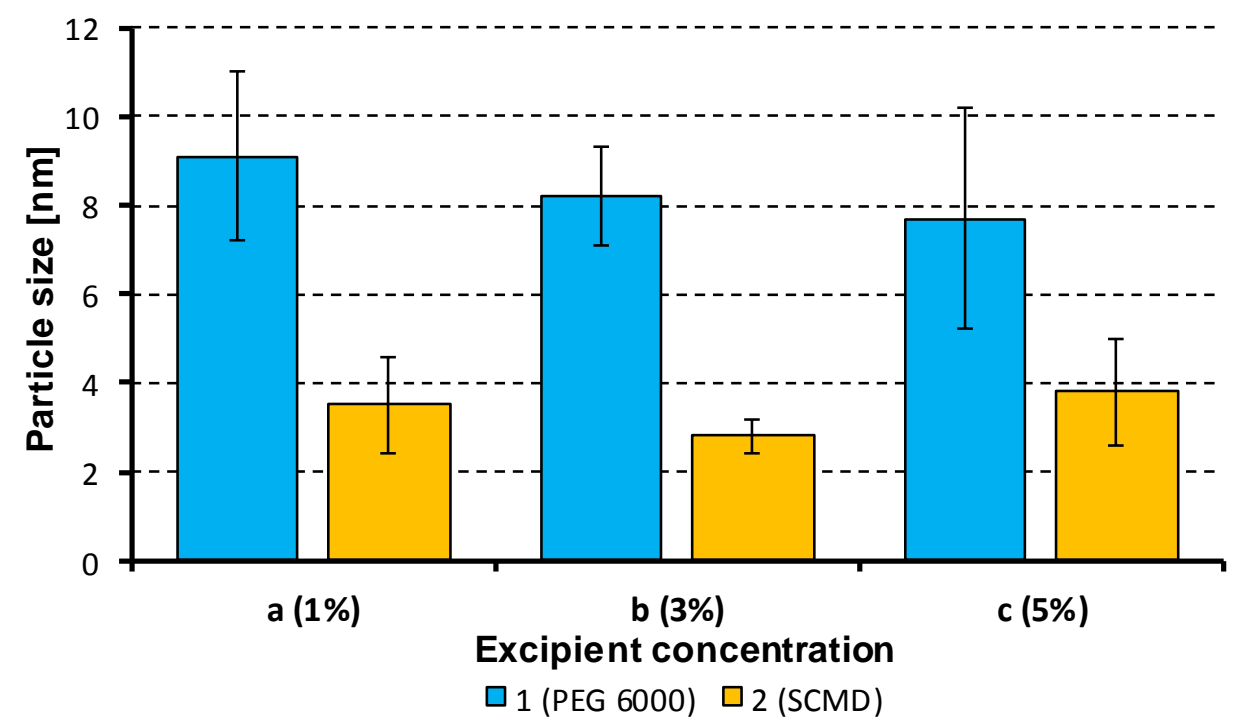

It can be concluded that the applied method can be used as an effective and affordable technique for preparation of nanoparticles. The selected conditions are convenient for formation of nanoparticles, and the used excipients are principally applicable as nanoparticle stabilizers. Nanoparticles of risedronate prepared in this manner can be subsequently used for nanoparticle formulations with supposed enhanced bioavailability.

\section{EXPERIMENTAL}

\section{General Procedure for Preparation of Nanoparticles}

Risedronate sodium and both excipients, macrogol 6000 (PEG) and sodium salt of carboxymethyl dextran (SCMD), were purchased from Sigma-Aldrich (Germany). All compounds were of analytical grade. $\mathrm{H}_{2} \mathrm{O}$-HPLC - Mili-Q Grade was used as a solvent of excipients. Each excipient $(0.1 \mathrm{~g}, 0.3 \mathrm{~g}$ or $0.5 \mathrm{~g})$ was dissolved in water $(10 \mathrm{~mL})$, and three solutions with mass concentrations $1 \%, 3 \%$ and $5 \%$ were prepared. Risedronate sodium $(0.1 \mathrm{~g})$ was dissolved in water Mili-Q $(10 \mathrm{~mL})$, i.e. $1 \%$ solutions were prepared. The solution of risedronate in water was slowly dropped $(2 \mathrm{~mL} / \mathrm{min})$ to the aqueous solutions of excipients that were stirred $(600 \mathrm{rpm})$. Then the system was stirred $(600 \mathrm{rpm})$ for $15 \mathrm{~min}$ at $25^{\circ} \mathrm{C}$, after which the mixtures were transferred to an ultrasonic bath in the fume chamber, where they were mixed again for 20 minutes for homogenization of the sample. Finally the solvent was evaporated to the third of the initial volume.

\section{Dynamic Light Scattering Measurements}

The particle size of the substance in all the final samples was evaluated by means of a Brookhaven dynamic light scattering system BI 9000 (Brookhaven Instruments Corporation, Holtsville, NY, USA) with a goniometer SM-200 and an argon gas laser (Lexel 95, used argon line $514.5 \mathrm{~nm}$ ). Scattered intensity was registered at the scattering angle $90^{\circ}$ and at the temperature of $25^{\circ} \mathrm{C}$. All samples were dispersed by ultrasonics and additionally filtered directly before the measurement through syringe filters with $0.45 \mu \mathrm{m}$ pore size to remove mechanical impurities. Particle size distribution can be obtained from the multiexponential 
time correlation function of a polydisperse sample. The respective methods rely on the inverse Laplace transformation. CONTIN algorithm was used for calculations of particle size spectra from the scattering data. Five independent measurements and calculations of the time correlation function were carried out for each surfactant and each scattering angle. All presented results are reported as medium value of these independent measurements. The results are summarized in Table 1 and illustrated in Figure 2.

\section{ACKNOWLEDGEMENTS}

This study was supported by the Czech Science Foundation - GACR P304/11/2246. This contribution was the result of implementation of the following project: Centre of excellence for security research, ITMS code: 26240120034, supported by the Research and Development Operational Programme funded by the ERDF. This paper was also supported by the Nanotechnology - the basis for international cooperation project, reg. No. CZ.1.07/2.3.00/20.0074 supported by the Operational Programme "Education for competitiveness" and financed by the Structural Funds and from the state budget of the Czech Republic.

\section{REFERENCES}

1. Rogers, M.J.; Xiong, X.; Brown, R.J.; Watts, D.J.; Russell, R.G.; Bayless, A.V.; Ebetino, F.H. Structure-activity relationships of new heterocycle-containing bisphosphonates as inhibitors of bone resorption and as inhibitors of growth of Dictyostelium discoideum amoebae. Mol. Pharmacol. 1995, 47, 398-402.

2. Rogers, M.J.; Gordon, S.; Benford, H.L.; Coxon, F.P.; Luckman, S.P.; Monkkonen, J.; Frith, J.C. Cellular and molecular mechanisms of action of bisphosphonates. Cancer 2000, 88, 2961-2978.

3. van Beek, E.R.; Lowik, C.W.; Ebetino, F.H.; Papapoulos, S.E. Binding and antiresorptive properties of heterocycle-containing bisphosphonate analogs: Structureactivity relationships. Bone 1998, 23, 437-442.

4. Aft, R.; Perez, J.R.; Raje, N.; Hirsh, V.; Saad, F. Could targeting bone delay cancer progression? Potential mechanisms of action of bisphosphonates. Crit. Rev. Oncol. Hematol. 2012, 82, 233-248.

5. Väänänen, H.K.; Zhao, H.; Mulari, M.; Halleen, M. The cell biology of osteoclasts function. J. Cell Sci. 2000, 113, 377-381.

6. Ezra, A.; Golomb, G. Administration routes and delivery systems of bisphosphonates for the treatment of bone resorption. Adv. Drug Del. Rev. 2000, 42, 175-195.

7. MedicineNet. http://www.medicinenet.com/risedronate/article.htm. Access on 7 October 2013.

8. eMedTV - Health Information Brought to Life ${ }^{\mathrm{TM}}$. http://osteoporosis.emedtv.com/. Access on 7 October 2013.

9. Drug Bank Online - drugbank.ca: http://www.drugbank.ca/drugs/DB00884. Access on 7 October 2013.

10. Mitchel, D.Y.; Barr, W.H.; Eusebio, R.A.; Pallone stevens, K.A.; Duke, F.P.; Rusell, D.A.; Nesbitt, J.D.; Powell, J.A.; Thompson, G.A. Risedronate pharmacokinetics intraand inter-subject variabiliy upno single-dose intravenous and oral administration. Pharm. Res. 2001, 18/2, 166-170.

11. Kerns, E.H.; Li, D. Drug-like Properties: Concept, Structure Design and Methods. Elsevier: San Diego, CA, USA, 2008. 
12. Dissette, V.; Bozzi, P.; Bignozzi, C.A.; Dalpiaz, A.; Ferraro, L.; Beggiato, S.; Leo, E.; Vighi, E.; Pasti, L. Particulate adducts based on sodium risedronate and titanium dioxide for the bioavailability enhancement of oral administered bisphosphonates. Eur. $J$. Pharm. Sci. 2010, 41, 328-336.

13. Kos, J.; Pentakova, M.; Oktabec, Z.; Krejcik, L.; Harokova, P., Hruskova, J.; Pekarek, T.; Dammer, O.; Havlicek, J.; Kral, V.; Dohnal, J.; Jampilek, J. Crystallization products of risedronate with carbohydrates and their substituted derivatives. Molecules 2011, 16, 3740-3760.

14. Konan, Y.N.; Berton, M.; Gurny, R.; Allemand, E. Enhanced photodynamic activity of meso-tetra(4-hydroxyphenyl)porphyrin by incorporation into sub-200 nm nanoparticles. Eur. J. Pharm. Sci 2003, 18, 241-249.

15. Bawa, R. Nanoparticle-based therapeutics in humans: A survey. Nanotech. Law Business 2008, 5, 135-155.

16. Bawa, R. Nanopharmaceuticals for drug delivery - A review. Drug Delivery 2009, 3, 122-127.

17. Vaculikova, E.; Grunwaldova, V.; Kral, V.; Dohnal, J.; Jampilek, J. Primary investigation of the preparation of nanoparticles by precipitation. Molecules 2012, 17, 11067-11078.

18. Vaculikova, E.; Grunwaldova, V.; Kral, V.; Dohnal, J.; Jampilek, J. Preparation of candesartan and atorvastatin nanoparticles by solvent evaporation. Molecules 2012, 17, $13221-13234$.

19. Merkus, H.G. Particle Size Measurements: Fundamentals, Practice, Quality. Springer Science+Business Media B.V.: Dordrecht, The Netherlands, 2009. 